\title{
An optical toolbox for total control of droplet microfluidics
}

\author{
Charles N. Baroud ${ }^{1}$, Matthieu Robert de Saint Vincent ${ }^{2}$, and Jean-Pierre Delville ${ }^{2}$ \\ ${ }^{1}$ LadHyX and Department of Mechanics, \\ Ecole Polytechnique, 91128 Palaiseau cedex, France \\ ${ }^{2}$ Université Bordeaux 1, CPMOH (UMR CNRS 5798) \\ 351 Cours de la Libération, F-33405 Talence cedex, France
}

April 27, 2007

\begin{abstract}
The use of microfluidic drops as microreactors hinges on the active control of certain fundamental operations, such as droplet formation, transport, division and fusion. Recent work has demonstrated that local heating from a focused laser can apply a thermocapillary force on a liquid interface sufficient to block the advance of a droplet in a microchannel (Baroud et al., Phys. Rev. E. V 75, p.046302). Here, we demonstrate the usefulness of this optical approach by implementing the operations mentioned above, without the need for any special microfabrication or moving parts. Building blocks such as a droplet valve, sorter, fuser, or divider are shown, as is the combination of a valve and fuser using a single laser spot.
\end{abstract}

\section{Introduction}

Droplets are natural candidates for use as microreactors, since they transport fluid with no dispersion and may be formed and manipulated using microfluidic techniques $[1,2,3,4]$. Indeed, a drop may be formed with a known composition and volume $[5,6,7]$ and transported by an inert fluid without loss of the solute species and without cross-contamination [8]. Furthermore, fusion of two drops containing two reactive species leads to the onset, on demand, of a reaction [9] whose product may be sampled by breaking the drop at a bifurcation [10]. Finally, logical operations can be performed on drops by sorting them based on a test of their contents, as they reach a bifurcation in the microchannel [11, 12]. The above operations form the basis of a droplet-based lab-on-a-chip which can be designed through an intelligent combination of a few building blocks. Conversely, a lack of active control over individual drops would severely limit the usefulness of droplet microreactors.

However, acting on individual drops in microchannels remains difficult. Recent publications have demonstrated, via electrode micropatterning on the chip, the use of electric fields to apply forces on droplets [11] or to merge them [9, 13]. However, the forces generated through dielectrophoresis were measured to be in the range of a few $\mathrm{nN}$ and scale with the cube of the drop radius, since the electrophoretic force is a body force [11]. This is a highly unfavourable scaling which implies that the force generated will quickly decrease as the drop size decreases. In contrast, others have demonstrated the use of surface forces to manipulate drops on open substrates by modulating their surface properties chemically, electrically or thermally (see e.g. Ref. [14] and references therein). These surface forces become dominant 
over body forces at small scales, as the ratio of surface to volume becomes large. It is natural therefore to look for a surface mechanism for the manipulation of drops inside the robust environment of a microchannel.

Along these lines, we have recently demonstrated that forces near the $\mu \mathrm{N}$ range could be produced on a droplet by optically heating a water-oil-surfactant interface with a laser wave [12]. This force is generated through the thermocapillary (or Marangoni) effect, by which the surface tension varies due to a temperature variation; localized heating from a focused laser therefore leads to a spatial imbalance of surface tension which, in turn, induces a flow inside and around the drop. By computing the shear and pressure fields associated with the external flow, one may find that a net force is produced on the drop [15]. A theoretical analysis for strongly localized heating shows a scaling that is highly favourable to miniaturization, since the total force is predicted to increase as the drop radius decreases [12].

In our experiments, we observe that the surface tension rises as the temperature is increased. This anomalous behaviour, likely due to the presence of surfactant at concentrations $[16,17]$, yields a force that pushes the drop away from the hot spot and acts to block it in the presence of an external carrier flow. Since the time required for the Marangoni flow to appear is short enough, a droplet can be blocked during its formation, corresponding to a contactless optical microfluidic valve, which can also be used to control the size of the drops thus produced. Finally, drops can also be sorted by simply illuminating one exit of a bifurcating microchannel [12].

Below, we show the generality of this optical approach and how it may be used to provide a complete set of tools for the manipulation of drops in microchannels. These tools allow the control of drop formation and sorting, as previously demonstrated, but also drop fusion and division. We also demonstrate how the operations may be combined, while still using a single laser spot, through a combination of channel geometry and laser actuation. This opens the way for total control of droplet microreactors without the need for specific microfabrication.

\section{Experimental setup}

Our experimental setup consists of a microchannel fabricated in Polydimethylsiloxane (PDMS), using standard soft lithography techniques. Water and oil (Hexadecane $+2 \% \mathrm{w} / \mathrm{w}$ Span 80, a surfactant) are pumped into the channel at constant flowrates, $Q_{\text {water }}$ and $Q_{\text {oil }}$, using glass syringes and syringe pumps; the fluid may also be pumped at constant pressure. Channel widths are in the range $100-500 \mu \mathrm{m}$ and the height is in the range $25-50 \mu \mathrm{m}$. Local heating is produced by focusing a continuous Argon-Ion laser (wavelength in vacuum $\lambda_{A r^{+}}=514 \mathrm{~nm}$ ), in the $\mathrm{TEM}_{00}$ mode through a microscope objective. The absorption of the laser is mediated by the addition of a dye, such as fluorescein $(0.1 \% \mathrm{w} / \mathrm{w})$, in the water phase. The resulting optical absorption of the aqueous phase is about $1.18 \mathrm{~cm}^{-1}$.

Different microscope objectives were used to focus the laser inside the microchannel, ranging from $\times 2.5$ to a $\times 10$ magnification, which correspond to beam waists $\left(\omega_{0}\right)$ in the range of 10.4 to $2.6 \mu \mathrm{m}$. The Fresnel length, defined as $L_{F}=n \pi \omega_{0}^{2} / \lambda$ where $n$ is the refractive index and $\lambda$ the wavelength in vacuum, may be estimated at $L_{F} \simeq 50 \mu \mathrm{m}$ by using $n=1.33$ and $\omega_{0}=2.5 \mu \mathrm{m}$. Consequently, we can assume that the focused beam is almost cylindrical over a distance of $100 \mu \mathrm{m}$ (50 $\mu \mathrm{m}$ on each side of the beam waist), which is twice the largest

thickness of our channels. This implies that the use of low magnification objectives makes the behavior rather insensitive to the exact focus plane, as opposed for example to laser tweezers. 


\section{Formation and fusion}

\subsection{Microfluidic valve}

The valve mechanism for two-phase flows was recently achieved [12] by illuminating the wateroil interface during the drop formation at a cross-junction, with a laser power $(P)$ on the order of a few tens of $\mathrm{mW}$, focused through a microscope objective. The local heating thus produced was shown to completely block the advance of the interface for a time $\tau_{b}$ which increased with increasing laser power. This blocking also provided control over the size of the drops thus produced, since they were inflated by the syringe pumps operating at a constant flowrate.

This valve is generic and works equally well in a $\mathrm{T}$ geometry where the oil and water arrive either from opposite channels (Fig 1a) or from perpendicular channels (e.g. Fig. 4). Similar blocking is also observed if the flows are driven at constant pressure or by mixing pressure and flowrate sources. For instance, Fig. 1a shows the laser blocking the drop shedding at different locations with the oil flow (bottom channel) driven at constant flowrate and the water flow (top channel) driven at constant pressure. In the absence of the laser, drops are formed in a periodic fashion. In the presence of the laser, the water interface is blocked at the laser focus, as shown in the figure, while the oil continues to flow. The variation of the blocking time $\tau_{b}$ with the laser power and position is illustrated in Fig. 1b. While $\tau_{b}$ increases approximately linearly with the power above an initial threshold, it also displays a dependence on the laser position in the microchannel. The values of the onset and the slope of $\tau_{b}$ depend on the details of the flow, but the same general behaviour is observed independently of the microchannel geometry, flowrates, or pumping method.

The dependence of the blocking time on beam waist was explored in a cross-geometry by keeping constant the fluid flow rates $\left(Q_{\text {water }}=0.12 \mu \mathrm{L} / \mathrm{min}\right.$ and $\left.Q_{\text {oil }}=0.3 \mu \mathrm{L} / \mathrm{min}\right)$ and the laser position. The geometry that was used corresponds to a cross-junction with oil channel widths $100 \mu \mathrm{m}$ and water channel width $200 \mu \mathrm{m}$. The laser was placed at a distance $200 \mu \mathrm{m}$ downstream of the oil channel centerline and the blocking time $\left(\tau_{b}\right)$ was measured as a function of beam waist, which was varied by changing the microscope objective. The measurements of $\tau_{b}$ were normalized by the natural emission frequency of the drops $\left(F_{0}\right)$ and were fitted by straight lines to determine the threshold power $\left(P_{t h}\right)$ and slope $\left(S=F_{0} d \tau_{b} / d P\right)$. While $P_{t h}$ was found to remain constant at $P_{t h} \simeq 40 \mathrm{~mW}, S$ increased with decreasing beam waist as $S=4.5 \times 10^{-3}, 8.2 \times 10^{-3}$ and $13.4 \times 10^{-3} \mathrm{~W}^{-1}$ for $\omega_{0}=10.3,5.2$ and $2.6 \mu \mathrm{m}$ respectively.

\subsection{Fusion of drops}

Fusing droplets is the step that allows chemical reactions by bringing together the reactants originally contained in separate drops. However, simply putting drops in contact is typically insufficient to induce merging, since a lubrication film between them prevents the water contained in the two drops from actually touching. Indeed, the presence of surfactant molecules on an oil-water interface is known to stabilize drops against merging [18, 19]. Localized heating close to the nearly touching interfaces may be used to evacuate the surfactant molecules and with them the oil film, as shown in Fig. 2. Here, the downstream drop is held stationary by the laser heating until a second one collides with it (Fig. 2a). At this point, the two drops advance until the laser gets near the adjacent interfaces, and we observe that the oil film is evacuated and the two drops rapidly merge.

Similar merging may be obtained in a long train of drops, as shown in Fig. 3. Here, a train of water drops is carried by an oil flow in a wide channel. Again, these drops are 

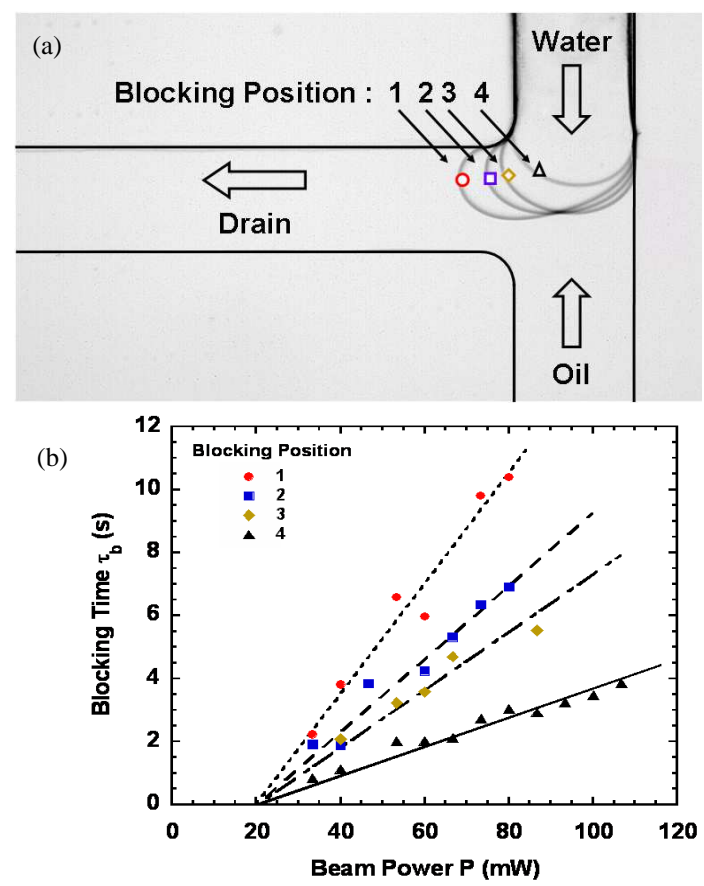

Figure 1: Microfluidic valve in a $\mathrm{T}$ geometry. (a) Superposition of microscopic images of the laser blocking the interface at different locations in the microchannel. All channels are $100 \mu \mathrm{m}$ wide. (b) Dependence of the blocking time $\tau_{b}$ on laser power and position (indicated in (a)) for $Q_{\text {oil }}=0.05 \mu \mathrm{L} / \mathrm{min}$ and $P_{\text {water }}=2.3 \times 10^{3} \mathrm{~Pa}, \omega_{0}=2.6 \mu \mathrm{m}$. The lines are linear fits to guide the eye.
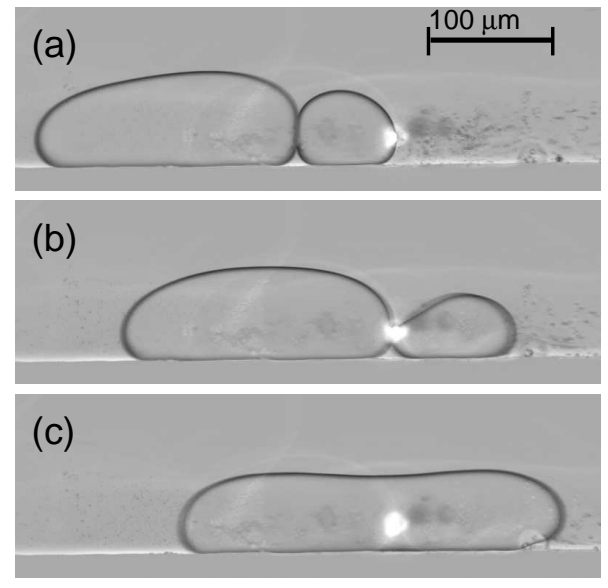

Figure 2: Time sequence showing droplet fusion through laser heating. (a) The blocking of a first drop by the laser brings the drop that follows in contact with it. (b) The two drops move forward together, their coalescence occurring when the beam reaches the touching interfaces, giving birth to a larger drop (c). Time between images is $40 \mathrm{~ms}$ and operating conditions are $Q_{\text {water }}=0.2 \mu \mathrm{L} / \mathrm{min}, Q_{\text {oil }}=0.9 \mu \mathrm{L} / \mathrm{min}, P=67 \mathrm{~mW}$ and $\omega_{0}=2.6 \mu \mathrm{m}$. 


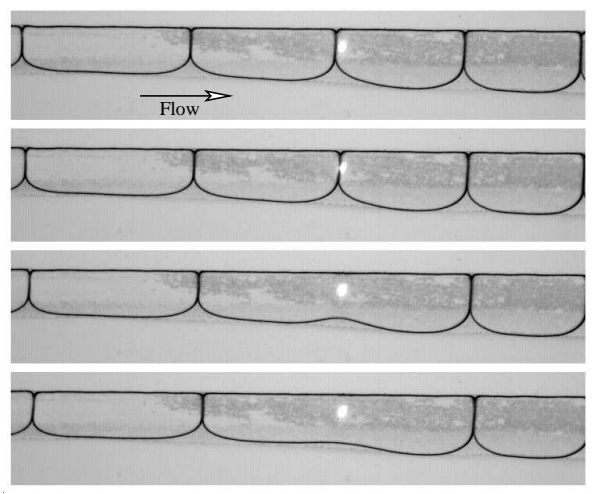

Figure 3: Localized fusion in a train of large drops. The drops, which flow from left to right, merge as the interface crosses the laser. Time between images is $30 \mathrm{~ms}$ and operating conditions are $Q_{\text {water }}=0.2 \mu \mathrm{L} / \mathrm{min}, Q_{\text {oil }}=0.3 \mu \mathrm{L} / \mathrm{min}, P=67 \mathrm{~mW}$ and $\omega_{0}=5.2 \mu \mathrm{m}$.

stable against merging due to the presence of the surfactant and spontaneous merging is never observed in our experiments (Figs. 3a,b). However, weak heating at the interface from the laser spot, although insufficient to block the drop advance, rapidly induces merging when the laser spot approaches the adjacent interfaces (Fig. 3c). Merging only occurs in the heated region (Fig. 3d) while the other interfaces remain unaffected. This shows that one may induce the merger of specific drops even in a complex flow which contains many drops and interfaces. A succession of such events is shown in the supporting video 1.

\subsection{Combined operations: Drop fusion at formation}

The synchronization of drops in order to combine their contents is a major challenge for lab-ona-chip operations. Alternating formation of drops from two sources was recently demonstrated by finely tuning the different water and oil flowrates [9]. This approach, however, is only useful in the simplest cases where only two droplet streams are involved and the downstream conditions are constant. A more robust approach would be for a downstream drop to delay its formation and wait for the upstream drop to catch up with it, at which point the two merge together. This corresponds, in our terms, to a combination of a valve and a fusion mechanism; once the two building blocks exist, combining them becomes a simple matter as shown in Fig. 4.

Here, drops are formed at successive T-junctions and flow down the same exit channel. In the absence of the laser forcing (not shown), the drop formation is not synchronized and neither do they merge if they do come into contact. The situation is different in the presence of the laser, which holds the downstream interface in place (Fig. 4a) until the upstream drop is formed and collides with it (Fig. 4b). Since the upstream drop completely blocks the channel, the hydrodynamic drag on the two-drop system becomes too large and the two drops start to flow again (Fig. 4c), merging together when their touching interfaces approach the laser (Fig. 4d). (See supporting video 2).

The valve and fusion actions here are performed with only one laser spot, showing how the different building blocks may be superposed by combining the laser action with a geometric constraint. This is done with no overhead in power or complexity with respect to a single operation, demonstrating how the technique may be scaled to a complex lab-on-a-chip 
involving many operations.

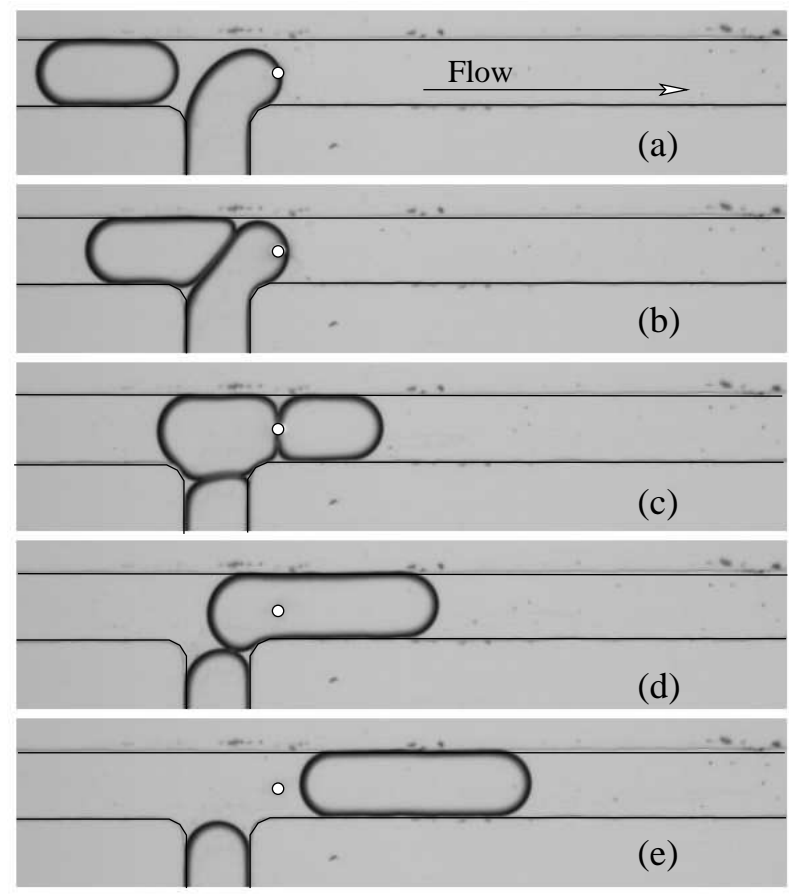

Figure 4: A forming drop is blocked by the laser-valve (a) until a second drop, formed upstream, collides with it (b). The collision liberates the front drop (c) and the two merge when their interfaces approach the laser(d). Operating conditions are $Q_{\text {water }}=0.1 \mu \mathrm{L} / \mathrm{min}$, $Q_{\text {oil }}=1 \mu \mathrm{L} / \mathrm{min}, P=67 \mathrm{~mW}$ and $\omega_{0}=5.2 \mu \mathrm{m}$. The laser position is represented by the white circle.

\section{Drop transport: division and sorting}

The remaining steps after the formation and merging of drops are their transport and division, which involve control over the route they follow at bifurcating channels. Two operations are demonstrated below: sampling a drop, i.e. dividing it into unequal daughter droplets of calibrated size, and sorting. A sampler which uses a combination of channel geometry and laser forcing is shown in Fig. 5. We see in it drops that are longer than the channel width and that arrive at a symmetric bifurcation, carried by the oil phase. At the bifurcation, the drops divide into two parts whose lengths in the daughter channels we label $L_{1}$ and $L_{2}$. We are interested in the ratio $\lambda \equiv\left\langle\left(L_{1}-L_{2}\right) /\left(L_{1}+L_{2}\right)\right\rangle$ which yields $\lambda=0$ for symmetric drops and $\lambda=1$ for complete sorting. The brackets $\langle\cdot\rangle$ here denote an average over several drops.

In the absence of the laser (Fig. 5a), we measure $\lambda=0.022 \pm 0.01$ for our microchannel, corresponding to a slight asymmetry in the microfabrication. When the laser is applied in front of one of the two exits, the water-oil interface is asymmetrically blocked at the laser position for a time $\tau_{b}$, while the other side continues to flow (Fig. 5b). After $\tau_{b}$, both sides of the drop continue forward into their respective channels, but the retardation of the right hand droplet tip produces an asymmetry in the breaking, measured by an increase in $\lambda$. Since the blocking time $\tau_{b}$ increases with the laser power, so does the asymmetry in the division, 
as shown in Fig. 5c. (see supporting video 3).

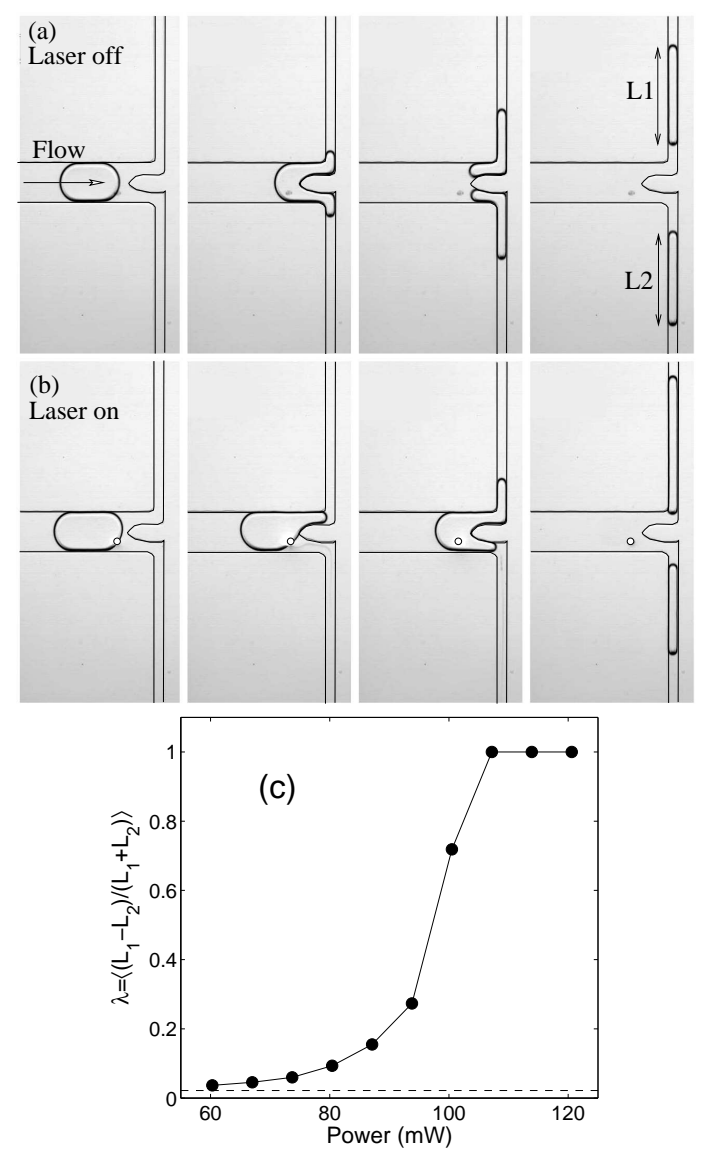

Figure 5: A droplet sampler: (a) Without laser forcing, a drop at a bifurcation divides into approximately equal daughter droplets. (b) By controlling the laser power (here $P=93 \mathrm{~mW}$ ), we control the pinning time of one side of the interface and thus the asymmetry of the division. Main channel width is $200 \mu \mathrm{m}$ and the time between images is $0.2 \mathrm{~s}$. Operating conditions are $Q_{\text {water }}=0.02 \mu \mathrm{L} / \mathrm{min}, Q_{\text {oil }}=0.2 \mu \mathrm{L} / \mathrm{min}$, and $\omega_{0}=5.2 \mu \mathrm{m}$. (c) Daughter droplet size dependence on laser power $(\lambda=0$ is for symmetric drops and $\lambda=1$ is for the sorter). The dashed line corresponds to the mean value of $\lambda$ in the absence of the laser.

Above a critical power (approximately $100 \mathrm{~mW}$ for the present configuration), the drop does not divide but is always diverted into the opposite branch. This sorting operation may be understood by considering the length of the droplet upstream of the laser: If the upstream length decreases below a critical size of approximately the channel width (corrected by the displacement of the laser with respect to the channel center), the drop takes a circular shape and loses contact with the right hand wall. In this case, a tunnel opens for the oil to flow between the drop and the wall and the drop does not divide anymore but is pushed into the left hand channel.

Unequal droplet splitting may be achieved through passive techniques, for example by varying the downstream resistance to the flow in simple cases $[10,20]$. However, the optical actuation adds an active component to the control of each droplet. It thus provides an additional control parameter that can be used in conjunction with passive control, independently 
of the downstream conditions or of the microsystem's complexity.

\section{Generality and optimization}

Our approach to controlling microfluidic droplets relies on all-optical techniques which have been greatly developed in recent years in connection with microfluidic devices $[21,22,23$, $24,25]$. Indeed, optical trapping has become a standard tool in biophysics [26] and holographic [22] and generalized phase contrast [25] methods now allow a single laser to be divided into many spots which can be independently manipulated. The application of beam division techniques to thermocapillary control should be relatively straight-forward and it will allow the parallel implementation of many independent building blocks in a complex network of channels. For instance, many valves, fusers, and sorters may be operated independently through the implementation of holographic division of the laser or by sweeping a single beam with a galvanometric mirror. Furthermore, a judicious choice of a laser wavelength and fluid combinations can improve the efficiency of the approach. In this regard, current experiments have reproduced the above results with an infrared laser which acts directly on the water molecules, allowing us to work without the need for an absorbing dye.

Moreover, the physical scaling laws for this forcing technique are favourable to further miniaturization, since the technique takes advantage of the dominance of surface effects in microfluidics. The force produced by the thermocapillary flow was theoretically found to scale as $1 / R$, where $R$ represents the in-plane radius of curvature of the drop at the hot spot [12]. The force is therefore expected to increase as the drop size decreases, as long as the local heating hypothesis may be maintained. This scaling may be used to optimize the performance of the system, for example by using channels with a variable width or by placing the laser at the position with highest drop curvature. Such optimization should allow the implementation of the devices with minimal laser power, further promoting parallelization and portability.

The response time should also scale favourably with miniaturization since it is limited by the heat and viscous diffusion processes and thermal inertia. The latter decreases as the cube of the length scale and is therefore negligibly small in microchannels. Moreover, the viscous diffusion time $\left(\tau_{\text {visc }} \sim \ell^{2} / \nu\right.$, where $\ell \simeq 30 \mu \mathrm{m}$ is a typical length scale and $\nu \simeq 10^{-6} \mathrm{~m}^{2} / \mathrm{s}$ is the fluid kinematic viscosity) and the thermal diffusion time $\left(\tau_{\text {th }} \sim \ell^{2} / D\right.$, where $D \simeq 10^{-7} \mathrm{~m}^{2} / \mathrm{s}$ is the thermal diffusion coefficient) are both on the order of a few ms, indicating that droplet manipulation at the $\mathrm{kHz}$ range may be possible.

Finally, since the method requires no moving parts or special microfabrication, the forcing is reconfigurable in real-time and may be adapted to many different microchannel geometries. Owing to its flexibility and scalability, our optical approach offers a complete toolbox for droplet based lab-on-a-chip applications.

\section{Acknowledgements}

Useful discussions are acknowledged with François Gallaire and Régis Wunenburger. This work was partially funded by the CNRS Projet interdisciplinaire de recherche "Microfluidique et Microsystèmes Fluidiques", the Conseil Régional d'Aquitaine (Université Bordeaux 1 group), and the convention X-DGA (Ecole Polytechnique group). 


\section{References}

[1] T.M. Squires and S.R. Quake. Microfluidics: Fluid physics at the nanoliter scale. Rev. Mod. Phys., 77(3):977-1026, July 2005.

[2] J. Atencia and D.J. Beebe. Controlled microfluidic interfaces. Nature, 437:648-655, 2005.

[3] A. Günther and K.F. Jensen. Multiphase microfluidics: from flow characteristics to chemical and material synthesis. Lab Chip, 6:1487-1503, 2006.

[4] H. Song, D.L. Chen, and R.F Ismagilov. Reactions in droplets in microfluidic channels. Angew. Chemie, int. ed., 45:7336-7356, 2006.

[5] T. Thorsen, R.W. Roberts, F.H. Arnold, and S.R. Quake. Dynamic pattern formation in a vesicle-generating microfluidic device. Phys. Rev. Lett., 86(18):4163-4166, 2001.

[6] S.L. Anna, N. Bontoux, and H.A. Stone. Formation of dispersions using 'flow-focusing' in microchannels. Appl. Phys. Lett., 82(3):364-366, 2003.

[7] R. Dreyfus, P. Tabeling, and H. Willaime. Ordered and discordered patterns in two phase flows in microchannels. Phys. Rev. Lett., 90:144505, April 2003.

[8] M. Chabert, K.D. Dorfman, P. de Cremoux, J. Roeraade, and J-L. Viovy. Automated microdroplet platform for sample manipulation and polymerase chain reaction. Anal. Chem., 78:7722-7728, 2006.

[9] K. Ahn, J. Agresti, H. Chong, M. Marquez, and D. Weitz. Electrocoalescence of drops synchronized by size-dependent flow in microfluidic channels. Appl. Phys. Lett., 88:264105, 2006.

[10] D.R. Link, S.L. Anna, D.A. Weitz, and H.A. Stone. Geometrically mediated breakup of drops in microfluidic devices. Phys. Rev. Lett., 92(5):054503, 2004.

[11] K. Ahn, C. Kerbage, T. Hynt, R.M. Westervelt, D.R. Link, and D. Weitz. Dielectrophoretic manipulation of drops for high-speed microfluidic sorting devices. Appl. Phys. Lett., 88:024104, 2006.

[12] Charles N. Baroud, Jean-Pierre Delville, Francois Gallaire, and Regis Wunenburger. Thermocapillary valve for droplet production and sorting. Physical Review E (Statistical, Nonlinear, and Soft Matter Physics), 75(4):046302, 2007.

[13] C. Priest, S. Herminghaus, and R. Seemann. Controlled electrocoalescence in microfluidics: Targeting a single lamella. Appl. Phys. Lett., 89:134101, Sept 2006.

[14] A.A. Darhuber and S.M. Troian. Principles of microfluidic actuation by modulation of surface stresses. Annu. Rev. Fluid Mech., 37:425-455, 2005.

[15] N.O. Young, J.S. Goldstein, and M.J. Block. The motion of bubbles in a vertical temperature gradient. J. Fluid Mech., 6:350-356, 1959.

[16] B. Berge, O. Konovalov, J. Lajzerowicz, A. Renault, J.P. Rieu, M. Vallade, J. AlsNielsen, G. Grübel, and J.F. Legrand. Melting of short 1-alcohol monolayers on water: Thermodynamics and x-ray scattering studies. Phys. Rev. Lett., 73(12):1652-1655, 1994. 
[17] E. Sloutskin, C.D. Bain, B.M. Ocko, and M. Deutsch. Surface freezing of chain molecules at the liquid-liquid and liquid-air interfaces. Faraday Discuss., 129:1-14, 2005.

[18] J. Bibette, D.C. Morse, T.A. Witten, and D.A. Weitz. Stability criteria for emulsions. Phys. Rev. Lett., 69:2439-2442, 1992.

[19] Y. Amarouchene, G. Cristobal, and H.. Kelay. Noncoalescing drops. Phys. Rev. Lett., 87:206104, 2001.

[20] L Ménétrier-Deremble and P Tabeling. Droplet breakup in microfluidic junctions of arbitrary angles. Phys. Rev. E, 74:035303, 2006.

[21] A. Terray, J. Oakey, and D.W.M. Marr. Microfluidic control using colloidal devices. Science, 296:1841-1844, 2002.

[22] D.G. Grier. A revolution in optical maniupulation. Nature, 424:810-816, Aug. 2003.

[23] M.P. MacDonald, G.C. Spalding, and K. Dholakia. Microfluidic sorting in an optical lattice. Nature, 426:421-424, 2003.

[24] D.R. Burnham and D. McGloin. Holographic optical trapping of aerosol droplets. Optics Express, 14(9):4176-4182, 2006.

[25] I.R. Perch-Nielsen, P.J. Rodrigo, C.A. Alonzo, and J. Glückstad. Autonomous and 3d real-time multi-beam manipulation in a microfluidic environment. Optics Express, 14(25):12199-12205, 2006.

[26] J. Guck, R. Ananthakrishnan, T.J. Moon, C.C. Cunningham, and J. Käs. The optical stretcher - a novel, noninvasive tool to manipulate biological materials. Biophys. J., 81:767-784, 2001. 